TRANSACTIONS OF THE

AMERICAN MATHEMATICAL SOCIETY

Volume 362, Number 2, February 2010, Pages 673-685

S 0002-9947(09)04809-0

Article electronically published on September 15, 2009

\title{
A DEGENERATE SOBOLEV INEQUALITY FOR A LARGE OPEN SET IN A HOMOGENEOUS SPACE
}

\author{
SCOTT RODNEY
}

\begin{abstract}
In current literature, existence results for degenerate elliptic equations with rough coefficients on a large open set $\Theta$ of a homogeneous space $(\Omega, d)$ have been demonstrated; see the paper by Gutierrez and Lanconelli (2003). These results require the assumption of a Sobolev inequality on $\Theta$ of the form

$$
\left\{\int_{\Theta}|w(x)|^{2 \sigma} d x\right\}^{\frac{1}{2 \sigma}} \leq C\left\{\int_{\Theta} \mathcal{Q}(x, \nabla w(x)) d x\right\}^{\frac{1}{2}},
$$

holding for $w \in \operatorname{Lip}_{0}(\Theta)$ and some $\sigma \in(1,2]$. However, it is unclear when such an inequality is valid, as techniques often yield only a local version of (1):

$$
\left\{\frac{1}{\left|B_{r}\right|} \int_{B_{r}}|v(x)|^{2 \sigma}\right\}^{\frac{1}{2 \sigma}} \leq C r\left\{\frac{1}{\left|B_{r}\right|} \int_{B_{r}} \mathcal{Q}(x, \nabla v(x)) d x+\frac{1}{\left|B_{r}\right|} \int_{B_{r}}|v(x)|^{2} d x\right\}^{\frac{1}{2}},
$$
holding for $v \in \operatorname{Lip}_{0}\left(B_{r}\right)$, with $\sigma$ as above. The main result of this work shows that the global Sobolev inequality (1) can be obtained from the local Sobolev inequality (2) provided standard regularity hypotheses are assumed with minimal restrictions on the quadratic form $\mathcal{Q}(x, \cdot)$. This is achieved via a new technique involving existence of weak solutions, with global estimates, to a 1-parameter family of Dirichlet problems on $\Theta$ and a maximum principle.
\end{abstract}

\section{INTRODUCTION}

This work addresses difficulties in obtaining existence and global estimates of weak solutions to second order linear subelliptic Dirichlet problems in divergence form with rough coefficients. Specifically, a local to global result for degenerate Sobolev inequalities is presented. This is achieved via a new process involving weak solutions, with global estimates, to a 1-parameter family of degenerate Dirichlet problems and a maximum principle.

Let $\Omega \Subset \mathbb{R}^{n}$ be open with $n \geq 3$. Denote by $\mathcal{Q}(x, \xi)=\xi^{\prime} Q(x) \xi$ a bounded, measurable, non-negative semi-definite quadratic form on $\Omega \times \mathbb{R}^{n}$. We assume that the Carnot-Carathéodory control distance $d$, defined by $\mathcal{Q}$ on $\Omega$, defines a homogeneous space structure in $\Omega$ in the sense of [15] and [12]. We consider equations of the form:

$$
X u=\nabla^{\prime} Q(x) \nabla u+H R u+S^{\prime} G u+F u=f+T^{\prime} g
$$

where the coefficient functions $H, G, g \in L_{l o c}^{q}(\Omega)$ and $F, f \in L_{l o c}^{q / 2}(\Omega)$ for $q>2 \sigma^{\prime}$, with $\frac{1}{\sigma}+\frac{1}{\sigma^{\prime}}=1$ where $\sigma$ is as in (12). The vector fields $R, S, T$ are assumed

Received by the editors October 1, 2007.

2000 Mathematics Subject Classification. Primary 35Hxx.

(c)2009 American Mathematical Society Reverts to public domain 28 years from publication 673 
to be subunit with respect to $\mathcal{Q}(x, \cdot)$ in $\Omega$. Recall that a vector field $V(x)=$ $\sum_{j=1}^{n} v_{j}(x) \frac{\partial}{\partial x_{j}}$, identified with the vector $v(x)=\left(v_{1}(x), \ldots, v_{n}(x)\right)$, is said to be subunit with respect to the quadratic form $\mathcal{Q}$ in $\Omega$ if

$$
(v(x) \cdot \xi)^{2} \leq \mathcal{Q}(x, \xi)
$$

for every $x \in \Omega$ and $\xi \in \mathbb{R}^{n}$. The operator $X$ is assumed to satisfy the following negativity condition analogous to that of [4] in the elliptic case, that is,

$$
\int_{\Omega}(F v-G S v) \leq 0
$$

for all $v \in C_{0}^{\infty}(\Omega)$ with $v \geq 0$ in $\Omega$.

Remark 1.1. The negativity condition is sufficient for existence of weak solutions to Dirichlet problems involving the operator $X$; see (3). Indeed, if one sets $H=$ $G=g=0$ and $F=c, c$ a constant, with $\mathcal{Q}$ the identity quadratic form on $\mathbb{R}^{n}$, then one obtains the elliptic equation

$$
\Delta u+c u=f .
$$

In this case the negativity condition becomes $c \leq 0$, which is known to be sufficient for existence of weak solutions to equations of this type; see 4.

In a subsequent paper the existence theory for Dirichlet problems of the form

$$
\begin{aligned}
X u & =f+T^{\prime} g \text { in } \Theta \\
u & =\phi \text { on } \partial \Theta
\end{aligned}
$$

where $\Theta \Subset \Omega$ is open will be treated. To obtain existence and uniqueness of weak solutions, weak in the sense of [5], the Fredholm Alternative will be used. Thus, we are required to show that the solution of the zero problem

$$
\begin{aligned}
X u & =0 \text { in } \Theta \\
u & =0 \text { on } \partial \Theta
\end{aligned}
$$

is unique and zero a.e. and so a maximum principle is needed. To obtain such a maximum principle it is sufficient to generalize the weak maximum principle of 44, the source of the idea for this work. The main ingredient for this generalization is a global Sobolev inequality on $\Theta$ of the form

$$
\left(\int_{\Theta}|w|^{2 \sigma}\right)^{\frac{1}{2 \sigma}} \leq C\left(\int_{\Theta} \mathcal{Q}(x, \nabla w)\right)^{\frac{1}{2}},
$$

holding for all $w \in \operatorname{Lip}_{0}(\Theta)$ for some $\sigma \in(1,2]$. In the case where $X$ is elliptic we have $\sigma=\frac{n}{n-2}$, the classical sharp Sobolev exponent. In the current literature one is most often provided with a local version of the Sobolev inequality:

$$
\left(\frac{1}{\left|B_{r}(x)\right|} \int_{B_{r}(x)}|w|^{2 \sigma}\right)^{\frac{1}{2 \sigma}} \leq C r\left(\frac{1}{\left|B_{r}(x)\right|} \int_{B_{r}(x)} \mathcal{Q}(y, \nabla w)\right)^{\frac{1}{2}},
$$

holding for all $w \in \operatorname{Lip}_{0}\left(B_{r}(x)\right), 0<r<\delta \operatorname{dist}(x, \partial \Omega)$, where $B_{r}(x)$ is the CarnotCarathéodory control ball of radius $r>0$. The constant $\delta$ appearing in (10) is due to difficulties arising from the degeneracy of the quadratic form $\mathcal{Q}$. Unfortunately, a naive partition of unity argument using inequality (10) does not give a Sobolev 
inequality of the form (9). Rather, one obtains a weaker form of inequality (9). This inequality, referred to as a global weak Sobolev inequality, is of the form

$$
\left(\int_{\Theta}|w|^{2 \sigma}\right)^{\frac{1}{2 \sigma}} \leq C\left\{\left(\int_{\Theta} \mathcal{Q}(x, \nabla w)\right)^{\frac{1}{2}}+\left(\int_{\Theta}|w|^{2}\right)^{\frac{1}{2}}\right\}
$$

holding for all $w \in \operatorname{Lip}_{0}(\Theta)$. Inequality (11) is insufficient, using the techniques of 4, for the goal of establishing existence of weak solutions to Dirichlet problems of the form (17), due to a breakdown in a generalization of the proof of the weak maximum principle of [4. In [12] it is shown that interior regularity of weak solutions to (7) is obtained via a weak local Sobolev inequality. It is assumed in [12, and shall be for the rest of this work, that $\exists \sigma \in(1,2]$ and $C, \delta>0$ such that if $0<r<\delta \operatorname{dist}(x, \partial \Omega)$, then

$$
\begin{aligned}
\left(\frac{1}{\left|B_{r}(x)\right|} \int_{B_{r}(x)}|w|^{2 \sigma}\right)^{\frac{1}{2 \sigma}} & \\
& \leq C\left\{r\left(\frac{1}{\left|B_{r}(x)\right|} \int_{B_{r}(x)} \mathcal{Q}(y, \nabla w)\right)^{\frac{1}{2}}+\left(\frac{1}{\left|B_{r}(x)\right|} \int_{B_{r}(x)}|w|^{2}\right)^{\frac{1}{2}}\right\}
\end{aligned}
$$

for all $w \in \operatorname{Lip}_{0}\left(B_{r}(x)\right)$ where $B_{r}(x)$ is the Carnot-Carathéodory control ball of radius $0<r<\delta \operatorname{dist}(x, \Omega)$. This inequality is weaker than inequality (10) since it is easily obtained from the local form (10). Inequality (10) is not unreasonable to assume, as such an inequality can be achieved via a sub-representation inequality; see [12]. To obtain a sub-representation inequality of the form considered in [12] in the case where $Q(x)$ is diagonal with coefficients $q_{j}(x)$, one must take suprema of the coefficients $q_{j}(x)$ in large boxes of girth which may be as large as $r\|Q\|_{\infty}$. Thus, these cubes may spill outside of the overlying set $\Omega$. The $\delta$ appearing above is, therefore, essentially $1 /\|Q\|_{\infty}$. See [12] for more details. Due to the weaker nature of inequality (12) compared with inequality (10), it will be the starting point of the work to follow.

It is the goal of this paper to show that the weak local Sobolev inequality (12) implies the global Sobolev inequality (9) on $\Theta$. This is achieved by solving a family of homogeneous Dirichlet problems related to the principal part of the operator $X$, see (21), provided that we assume an $L^{2}-L^{2}$ Poincaré inequality, see below. The Sobolev inequality on the set $\Theta$ allows one to conclude existence and global estimates for weak solutions of the homogeneous Dirichlet problem (7), to be dealt with in subsequent work. This demonstrates the close connection between Sobolev inequalities and existence of weak solutions to Dirichlet problems also evident in the elliptic case.

The Poincaré Inequality: $\exists C_{p}>0$ such that for $x \in \Omega$ and $0<r<\delta \operatorname{dist}(x \partial \Omega)$ we have the Poincaré inequality:

$$
\left(\frac{1}{\left|B_{r}(x)\right|} \int_{B_{r}(x)}\left|w-w_{B_{r}}\right|^{2}\right)^{\frac{1}{2}} \leq C_{p} r\left(\frac{1}{\left|B_{r}(x)\right|} \int_{B_{r}(x)} \mathcal{Q}(y, \nabla w)\right)^{\frac{1}{2}}
$$

holding for all $w \in \operatorname{Lip}\left(B_{r}(x)\right)$.

Lastly, the setting in which the following takes place is the Hilbert space $Q H_{0}^{1}(\Theta)$ $=W_{Q}^{1,2}(\Theta)$. Due to the degeneracy of the quadratic form $\mathcal{Q}$, the gradient of an 
element $u \in Q H_{0}^{1}(\Theta)$ may not be uniquely determined by its projection onto $L^{2}$. This strange behavior does not have strong implications for this work but does play a role in regularity theory; see 13 .

To define the degenerate Sobolev space $Q H_{0}^{1}(\Theta)$ for an open bounded subset $\Theta$ of the homogeneous space $(\Omega, d)$, we first define the related space $\mathcal{L}^{2}(\Theta, \mathcal{Q})$. The following development of the space $\mathcal{L}^{2}(\Theta, \mathcal{Q})$ is due to E. Sawyer and R.L. Wheeden in [13. Define the form weighted vector space $\mathcal{L}^{2}(\Theta, \mathcal{Q})$ to consist of all measurable vector valued functions $h(x)=\left(h_{1}(x), \ldots, h_{n}(x)\right)$ defined a.e. on $\Theta$ for which

$$
\|h\|_{\mathcal{L}^{2}(\Theta, \mathcal{Q})}=\left(\int_{\Theta} \mathcal{Q}(x, h(x)) d x\right)^{\frac{1}{2}}<\infty .
$$

If one identifies two functions $h, g \in \mathcal{L}^{2}(\Theta, \mathcal{Q})$ if $\|h-g\|_{\mathcal{L}^{2}(\Theta, \mathcal{Q})}=0$, then (14) defines a norm on the vector space $\mathcal{L}^{2}(\Theta, \mathcal{Q})$. As is shown in [13], with the norm (14) $\mathcal{L}^{2}(\Theta, \mathcal{Q})$ is a Hilbert space with respect to the inner product

$$
<h, g>_{\mathcal{L}^{2}(\Theta, \mathcal{Q})}=\int_{\Theta} h(x)^{\prime} Q(x) g(x) d x .
$$

It should be noted that due to the above formulation the calculus extends to these spaces as can be seen in 13 and with a different approach in 7]. The author would like to thank E. Sawyer and R. Wheeden for their precise definition of the space $\mathcal{L}^{2}(\Theta, \mathcal{Q})$.

We now define the degenerate Sobolev space $Q H_{0}^{1}(\Theta)=W_{\mathcal{Q}}^{1,2}(\Theta)$. Consider the inner product on $\operatorname{Lip}_{0}(\Theta)$ given by

$$
(w, v)_{Q}=\int_{\Theta} w(x) v(x) d x+<\nabla w, \nabla v>_{\mathcal{L}^{2}(\Theta, \mathcal{Q})} .
$$

This inner product generates a norm on $\operatorname{Lip}_{0}(\Theta)$ given by

$$
\|w\|_{Q H_{0}^{1}(\Theta)}=(w, w)_{Q}^{\frac{1}{2}}=\left\{\|w\|_{2}+\|\nabla w\|_{\mathcal{L}^{2}(\Theta, \mathcal{Q})}\right\}^{\frac{1}{2}} .
$$

We define the space $Q H_{0}^{1}(\Theta)=W_{Q}^{1,2}(\Theta)$ as the completion of $\operatorname{Lip}_{0}(\Theta)$ under the norm (17). $Q H_{0}^{1}(\Theta)$ is then a Hilbert space with respect to the inner product (16); see [7] for more details.

The space $Q H_{0}^{1}(\Theta)$ consists of equivalence classes of Cauchy sequences of Lipschitz functions. The equivalence class represented by a Cauchy sequence $\left\{u_{j}\right\} \subset$ $\operatorname{Lip}_{0}(\Theta)$ will be denoted by $\left[\left\{u_{j}\right\}\right]$. Due to the nature of the norm (17), if $\left\{w_{j}\right\} \subset$ $\operatorname{Lip}_{0}(\Theta)$ is Cauchy with respect to (17), then $\left\{w_{j}\right\}$ is also Cauchy in $L^{2}(\Theta)$ and $\left\{\nabla w_{j}\right\}$ is Cauchy in $\mathcal{L}^{2}(\Theta, \mathcal{Q})$. Thus for the equivalence class $\left[\left\{w_{j}\right\}\right]$ there are unique functions $w_{L^{2}} \in L^{2}(\Theta), w_{\nabla} \in \mathcal{L}^{2}(\Theta, \mathcal{Q})$ associated to it. As discussed in 13. this reasoning gives rise to a Hilbert space isomorphism $\mathcal{J}$ between $Q H_{0}^{1}(\Theta)$ and a closed subspace of $L^{2}(\Theta) \times \mathcal{L}^{2}(\Theta, \mathcal{Q})$ denoted by $\mathcal{W}_{Q}^{1,2}(\Theta)$. This Hilbert space isomorphism is defined by

$$
\mathcal{J}\left(\left[\left\{u_{j}\right\}\right]\right)=\left(u_{L^{2}}, u_{\nabla}\right)
$$

Remark 1.2. The gradient of a function $u \in Q H_{0}^{1}(\Theta)$ is a member of the space $\mathcal{L}^{2}(\Theta, \mathcal{Q})$. Due to the isomorphism $\mathcal{J}$ of $Q H_{0}^{1}(\Theta)$ into $\mathcal{W}_{Q}^{1,2}(\Theta)$ one identifies $u=$ $\left(u_{L^{2}}, u_{\nabla}\right)$. One may be tempted to believe that $u_{\nabla}$ is uniquely determined by the " $L^{2}$-part" of $u, u_{L^{2}}$. As can be seen in an example due to Fabes, Kenig and Serapioni in [1, this is in fact not the case. This does not affect the work to be presented here, but we shall need to consider the natural map $I: Q H_{0}^{1}(\Theta) \rightarrow L^{2}(\Theta)$ 
defined as $I=P \circ \mathcal{J}$, where $P$ is projection of $\mathcal{W}_{Q}^{1,2}(\Theta)$ onto the first component, such that

$$
I\left(\left[\left\{u_{j}\right\}\right]\right)=u_{L^{2}} .
$$

Due to the example of Fabes, Kenig and Serapioni, the natural map may not be one to one; however, as will be seen below, $I$ is a compact mapping, which is the essential property to be exploited here.

Some care has been taken to make a distinction between an equivalence class $\left[\left\{u_{j}\right\}\right]$ of $Q H_{0}^{1}(\Theta)$ and a pair $\left(u_{L^{2}}, u_{\nabla}\right) \in \mathcal{W}_{Q}^{1,2}(\Theta)$. However, what follows will contain some abuses of notation to simplify equations and inequalities. Indeed, $u$ will be used to denote an element $\left[\left\{u_{j}\right\}\right] \in Q H^{1}(\Theta)$, and this same $u$ will also be used to denote its $L^{2}$-part $u_{L^{2}}$. The difference will be taken as understood in the context presented. Further, by the definitions given above the Sobolev inequality (12) extends by density to $Q H_{0}^{1}\left(B_{r}\right)$ functions. The quadratic form $\mathcal{Q}$ also gives rise to a pointwise inner product and norm which are useful for simplifying notation. For $v, w \in \mathcal{L}^{2}(\Theta, \mathcal{Q})$ we write $\left\langle v, w>_{Q}=v^{\prime} Q(x) w\right.$ and the pointwise norm generated by this inner product is denoted by $\|w\|_{Q}^{2}=w^{\prime} Q(x) w$. These elements are well defined, as per [13] and [7, as $L^{1}(\Theta)$ functions.

The main theorems to be demonstrated are as follows.

Theorem 1.1. Let $(\Omega, d)$ be a homogeneous space, $\Theta \Subset \Omega$ an open set and $\mathcal{Q}(x, \cdot)$ a bounded, measurable, non-negative semi-definite quadratic form in $\Omega$. Then $\exists C=$ $C(\sigma, \Theta, d)$ so that the global Sobolev inequality

$$
\left(\frac{1}{|\Theta|} \int_{\Theta}|w|^{2 \sigma}\right)^{\frac{1}{2 \sigma}} \leq C\left(\frac{1}{|\Theta|} \int_{\Theta} \mathcal{Q}(x, \nabla w)\right)^{\frac{1}{2}}
$$

holds for all $w \in$ Lip $_{0}(\Theta)$ provided the following conditions hold:

1. the weak local Sobolev inequality (12);

2. the Poincaré inequality (13).

The main obstacle to proving theorem (1.1) is a weak maximum principle requiring the following compactness result concerning the natural map $I: Q H_{0}^{1}(\Theta) \rightarrow$ $L^{2}(\Theta)$, defined by $I(u)=u$. This result is similar to theorem 3.4 in $[3]$ but does not require the context of Lipschitz vector fields.

Theorem 1.2. Let $\Theta \Subset \Omega$ be open with $(\Omega, d)$ a homogeneous space. Let the quadratic form $\mathcal{Q}(x, \cdot)$ be as in Theorem (1.1). Then the natural map $I: Q H_{0}^{1}(\Theta) \rightarrow$ $L^{2}(\Theta)$ is compact provided the local Poincaré inequality (13) holds in $\Omega$.

Theorem (1.2) evolved substantially through discussion of 3 with S. K. Choi of the Singapore National University and R. Wheeden of Rutgers University and the author thanks them for their comments. Theorem (1.2) can be shown to hold in a much broader context and is the subject of current work joint with the authors just mentioned. This work may be found in the near future under the title "A Compactness Theorem for Generalized Sobolev Spaces".

The last step in obtaining the strong Sobolev inequality (9) is to exploit the close connection between the existence of weak solutions to a family of Dirichlet problems related to the principal part of the operator $X$ and Sobolev inequalities. 
Corollary 1.1. Let $0<\tau<1$ be given and let $q>2 \sigma^{\prime}$ where $\sigma$ is as in (12). If the local weak Sobolev inequality (12) holds in $\Omega$ and $f \in L^{\frac{q}{2}}(\Theta)$, then there exists a weak solution of the homogeneous Dirichlet problem

$$
\begin{aligned}
\nabla^{\prime} Q(x) \nabla u-\tau u & =f \text { in } \Theta \\
u & =0 \text { on } \partial \Theta .
\end{aligned}
$$

Further, if $\|f\|_{q / 2}=1, \exists C=C(\sigma, q, \Theta, d)>0$ so that this weak solution satisfies the global estimate

$$
\sup _{\Theta}|u| \leq C
$$

where $C$ is independent of $\tau$.

Theorem 1.1 will be achieved via Corollary 1.1, using the fact that the constant in (22) is independent of $\tau>0$ in a slight modification of the proof of the local Sobolev inequality appearing in [12, which was achieved via $L^{q}$-subellipticity for the homogeneous Dirichlet problem.

\section{ProOfS}

Proof of Theorem 1.2. Let $\left\{f_{n}\right\}$ be a sequence in the unit ball of $Q H_{0}^{1}(\Theta)$. Then from the definition of the space $Q H_{0}^{1}(\Theta)$ we have that $\left\{f_{n}\right\}$ is a bounded sequence in $L^{2}(\Theta)$ and so contains a weakly convergent subsequence in $L^{2}(\Theta)$ which we relabel as $\left\{f_{n}\right\}$. Our goal is to show that $\left\{f_{n}\right\}$ is Cauchy in $L^{2}(\Theta)$ giving that $\left\{f_{n}\right\}$ converges in norm. Let $\epsilon>0$ be given and let $0<r<\delta \operatorname{dist}(\Theta, \Omega), r$ will be chosen precisely in a moment. Cover $\Theta$ with $N=N(\epsilon)$ metric balls $\left\{B_{r}^{j}\right\}$ satisfying

$$
\begin{aligned}
& \text { (i) } \quad \Theta \subset \cup_{j=0}^{N} B_{r}^{j}, \\
& \text { (ii) } \quad \sum_{j=0}^{N} \chi_{B_{r}^{j}}(x) \leq M \text { for every } x \in \Omega .
\end{aligned}
$$

Note that the constant $M$ may be chosen to depend only on the quasimetric $d$ and not on $N$ or $\epsilon$. With this we have,

$$
\begin{aligned}
\left\|f_{n}-f_{m}\right\|_{2}^{2} & \leq \sum_{j=0}^{N} \int_{B_{r}^{j}}\left|f_{n}-f_{m}\right|^{2} d x \\
& =\sum_{j=0}^{N} \int_{B_{r}^{j}}\left|f_{n}-f_{m}-\left(f_{n}-f_{m}\right)_{B_{r}^{j}}+\left(f_{n}-f_{m}\right)_{B_{r}^{j}}\right|^{2} d x \\
& \leq \sum_{j=0}^{N} \int_{B_{r}^{j}}\left|f_{n}-f_{m}-\left(f_{n}-f_{m}\right)_{B_{r}^{j}}\right|^{2} d x+\sum_{j=0}^{N} \int_{B_{r}^{j}}\left|\left(f_{n}-f_{m}\right)_{B_{r}^{j}}\right|^{2} d x \\
& =I+I I .
\end{aligned}
$$

From the Poincaré inequality (13) and (ii) above we have,

$$
\begin{aligned}
I & \leq C_{p} r^{2} \sum_{j=0}^{N} \int_{B_{r}^{j}}\left|\left(\nabla\left(f_{n}-f_{m}\right)\right)^{\prime} Q(x) \nabla\left(f_{n}-f_{m}\right)\right|^{2} \\
& \leq C r^{2} .
\end{aligned}
$$


Thus, we may fix $\mathrm{r}$ so that $0<r<\delta \operatorname{dist}(\Theta, \Omega)$ and $I \leq \epsilon$. With this value of $\mathrm{r}$ we have

$$
\begin{aligned}
I I & \leq \sum_{j=0}^{N}\left|B_{r}^{j}\right|^{-1}\left|\int_{B_{r}^{j}}\left(f_{n}-f_{m}\right) d x\right|^{2} \\
& \leq \sum_{j=0}^{N}\left|B_{r}^{j}\right|^{-1}\left|\int_{\Theta}\left(f_{n}-f_{m}\right) \chi_{B_{r}^{j}} d x\right|^{2} .
\end{aligned}
$$

Since $\chi_{B_{r}^{j}} \in L^{2}(\Omega)$ as $\Omega \Subset \mathbb{R}^{n}$ we may choose, using the weak convergence of $\left\{f_{n}\right\}$, $m, n$ sufficiently large so that

$$
I I \leq \epsilon
$$

Thus $\left\{f_{n}\right\}$ is Cauchy in $L^{2}(\Theta)$. Therefore, any bounded sequence in $Q H_{0}^{1}(\Theta)$ contains a subsequence convergent in $L^{2}(\Theta)$ giving that $I$ is indeed a compact mapping.

Proof of Corollary 1.1 To begin, we obtain a weak Sobolev inequality on the set $\Theta$ via a standard partition of unity argument. The proof of the following lemma is omitted here as it is elementary.

Lemma 2.1. Let $\Theta$ be an open set with $\Theta \Subset \Omega$ and assume that the local weak Sobolev inequality (12) holds in $\Omega$. Then $\exists C=C(\sigma, \Theta)>0$ such that

$$
\left(\frac{1}{|\Theta|} \int_{\Theta}|w|^{2 \sigma}\right)^{\frac{1}{2 \sigma}} \leq C\left\{\left(\frac{1}{|\Theta|} \int_{\Theta} \nabla^{\prime} w Q(x) \nabla w\right)^{\frac{1}{2}}+\left(\frac{1}{|\Theta|} \int_{\Theta}|w|^{2}\right)^{\frac{1}{2}}\right\}
$$

for any $w \in \operatorname{Lip}_{0}(\Theta)$.

Consider the operator associated to $L=\nabla^{\prime} Q(x) \nabla$ given by $L_{\tau} u=\nabla^{\prime} Q(x) \nabla u-$ $\tau u$ and the associated Dirichlet problem

$$
\begin{aligned}
L_{\tau} u & =f \text { in } \Theta \\
u & =0 \text { on } \partial \Theta .
\end{aligned}
$$

By assumption, $f \in L^{q / 2}(\Theta)$ for some $q>2 \sigma^{\prime}$, $\sigma^{\prime}$ being the dual of the exponent $\sigma$ appearing in (12). The associated bi-linear form for this Dirichlet problem acting on $Q H_{0}^{1}(\Theta) \times Q H_{0}^{1}(\Theta)$ is given by:

$$
\mathcal{L}_{\tau}(u, v)=\int_{\Theta}<\nabla u, \nabla v>_{Q}+\tau \int_{\Theta} u v .
$$

Thus, a weak solution of (27) must then satisfy:

$$
\mathcal{L}_{\tau}(u, v)=-\int_{\Theta} f v
$$

for any $v \in Q H_{0}^{1}(\Theta)$. The linear functional acting on $Q H_{0}^{1}(\Theta)$ given by $\Phi(v)=$ $-\int_{\Theta} f v$ is clearly continuous since $\|f\|_{2} \leq C\|f\|_{q / 2}$ and $\|v\|_{2} \leq\|v\|_{Q H^{1}(\Theta)}$. Further, the bi-linear form $\mathcal{L}_{\tau}$ is both bounded and coercive on $Q H_{0}^{1}(\Theta)$. Indeed, the 
pointwise inequality $<\nabla u, \nabla v>_{Q} \leq\|\nabla u\|_{Q}\|\nabla v\|_{Q}$, holding almost everywhere in $\Theta$, together with Hölder's inequality gives

$$
\begin{aligned}
\mathcal{L}_{\tau}(u, v) & \leq \int_{\Theta}\|u\|_{Q}\|v\|_{Q}+\|u\|_{2}\|v\|_{2} \\
& \leq\left(\int_{\Theta}\|\nabla u\|_{Q}^{2}\right)^{1 / 2}\left(\int_{\Theta}\|\nabla v\|_{Q}^{2}\right)^{1 / 2}+\|u\|_{2}\|v\|_{2} \\
& \leq 2\|u\|_{Q H^{1}(\Theta)}\|v\|_{Q H^{1}(\Theta)}
\end{aligned}
$$

and hence boundedness of $\mathcal{L}_{\tau}$. The coercivity of $\mathcal{L}_{\tau}$ is immediate since $0<\tau<1$. Indeed,

$$
\begin{aligned}
\tau\|u\|_{Q H^{1}(\Theta)}^{2} & =\tau \int_{\Theta}\|\nabla u\|_{Q}^{2}+\tau\|u\|_{2}^{2} \\
& \leq \int_{\Theta}\|\nabla u\|_{Q}^{2}+\tau\|u\|_{2}^{2} \\
& =\mathcal{L}_{\tau}(u, u) .
\end{aligned}
$$

Therefore, by the Lax-Milgram theorem there exists a unique weak solution to (27).

For the second part of the theorem we follow the method of proof outlined in Theorem 8.15 of [4]; this argument also appears in [5]. If $u$ is a weak solution to (27), then by definition $u \in Q H_{0}^{1}(B)$. It is clear that we then have $u^{+} \in Q H_{0}^{1}(\Theta)$, where $u^{+}$is defined as in [7, so that $u^{+}$is a valid test function for $u$, i.e.

$$
\int_{\Theta}\left(\nabla u^{+}\right)^{\prime} Q \nabla u=-\int_{\Theta}\left(f u^{+}\right) .
$$

This equality is key and should be kept in mind for what will follow. Set $w=u^{+}+1$. For $\beta \geq 1$ we define, for $N>1, H_{N}:[1, \infty) \rightarrow \mathbb{R}_{+}$by

$$
H_{N}(s)= \begin{cases}0 & \text { if } s<1 \\ s^{\beta}-1 & \text { if } 1 \leq s \leq N \\ \beta N^{\beta-1}(s-N)+N^{\beta}-1 & \text { if } s>N .\end{cases}
$$

This function is clearly $C^{1}([1, \infty))$ with $H_{N}^{\prime} \in L^{\infty}$ and $H_{N}(1)=0$. The lemma on admissible compositions in [12] and [7] then gives $H_{N}(w) \in Q H_{0}^{1}(\Theta)$. Next, define the test function $G_{N}:[1, \infty) \rightarrow \mathbb{R}_{+}$by

$$
G_{N}(x)=\int_{1}^{x}\left(H_{N}^{\prime}(s)\right)^{2} d s,
$$

which satisfies the key inequality

$$
G_{N}(x) \leq x G_{N}^{\prime}(x) .
$$

We require that $G_{N} \in Q H_{0}^{1}(\Theta)$ in order to be able to test the weak solution against it. However, since $G_{N}(1)=0$ we have by the lemma on admissible compositions in [12] and [7] that indeed $G(w) \in Q H_{0}^{1}(\Theta)$. The weak Sobolev inequality 
(26) then gives:

$$
\begin{aligned}
\frac{1}{|\Theta|^{\frac{1}{\sigma}}}\left(\int_{\Theta}\left|H_{N}(w)\right|^{2 \sigma}\right)^{\frac{1}{\sigma}} & \leq \frac{C^{2}}{|\Theta|}\left(\int_{\Theta}\left\|\nabla H_{N}(w)\right\|_{Q}^{2}+\left\|H_{N}(w)\right\|_{2}^{2}\right) \text { by (26) } \\
= & \frac{C^{2}}{|\Theta|}\left(\int_{\Theta}\left(H_{N}^{\prime}(w)\right)^{2}(\nabla w)^{\prime} Q \nabla w+\left\|H_{N}(w)\right\|_{2}^{2}\right) \\
& =\frac{C^{2}}{|\Theta|}\left(\int_{\Theta}\left(\nabla G_{N}(w)\right)^{\prime} Q \nabla u+\left\|H_{N}(w)\right\|_{2}^{2}\right) .
\end{aligned}
$$

The last equality is due to the fact that $\nabla w=\nabla u^{+}=\nabla u$ where $\nabla u^{+} \neq 0$ and if $u^{+}=0$ we have that $w=1$, giving $G_{N}(w)=0$. Thus, using the fact that $u$ is a weak solution to (27),

$$
\frac{1}{|\Theta|^{\frac{1}{\sigma}}}\left(\int_{\Theta}\left|H_{N}(w)\right|^{2 \sigma}\right)^{\frac{1}{\sigma}} \leq \frac{C^{2}}{|\Theta|}\left(\int_{\Theta}|f| G_{N}(w)+\left.(1-\tau)|| H_{N}(w)\right|_{2} ^{2}\right) .
$$

Since $w \geq 1$, taking limits in all of the above we have by (30) and (33) that

$$
\begin{aligned}
\left(\int_{\Theta}\left|H_{N}(w)\right|^{2 \sigma}\right)^{\frac{1}{\sigma}} & \leq \frac{C^{2}}{|\Theta|^{\frac{\sigma-1}{\sigma}}}\left(\int_{\Theta}|f| w^{2} G_{N}^{\prime}(w)+(1-\tau)|| H_{N}(w) \|_{2}^{2}\right) \\
& =\frac{C^{2}}{|\Theta|^{\frac{\sigma-1}{\sigma}}}\left(\int_{\Theta}|f|\left(w H_{N}^{\prime}(w)\right)^{2}+\left\|H_{N}(w)\right\|_{2}^{2}\right) .
\end{aligned}
$$

Now $H_{N}$ and $H_{N}^{\prime}$ are increasing in $N$ and (34) holds for every $N$. The monotone convergence theorem applied to each side of the equation then yields:

$$
\begin{aligned}
\left\|w^{\beta}-1\right\|_{2 \sigma}^{2} & \leq \frac{C^{2}}{|\Theta|^{\frac{\sigma-1}{\sigma}}}\left(\int_{\Theta}|f|\left(w \cdot \beta w^{\beta-1}\right)^{2}+\left\|w^{\beta}-1\right\|_{2}^{2}\right) \\
& \leq C(\Theta)\left(\beta^{2}\left\|w^{2 \beta}\right\|_{\frac{q}{q-2}}+\beta^{2}\left\|w^{2 \beta}\right\|_{\frac{q}{q-2}}\right),
\end{aligned}
$$

since $w \geq 1$ and $1<\frac{q}{q-2}$ due to the choice of $q>2 \sigma^{\prime}$. Taking square roots and using the homogeneity of the $L^{p}$ norm, we obtain the reverse Hölder estimate:

$$
\left\|w^{\beta}\right\|_{2 \sigma} \leq M \beta\left\|w^{\beta}\right\|_{\frac{2 q}{q-2}}
$$

where $M=M(\Theta)$. Setting $\chi=\frac{\sigma(q-2)}{q}>1$, since $q>2 \sigma^{\prime}$, we rewrite the above as

$$
\|w\|_{\beta \chi q^{*}} \leq(M \beta)^{\frac{1}{\beta}}\|w\|_{\beta q^{*}}
$$

where $q^{*}=\frac{2 q}{q-2}$. Hence, (36) says that $w \in L^{\beta q^{*}}$ implies $w \in L^{\beta \chi q^{*}}$ which is a stronger inclusion since $\chi>1$. Following the argument found in [5] Theorem 3.1 we obtain:

$$
\sup _{y \in \Theta} u^{+} \leq \sup _{y \in \Theta} w \leq C(\sigma, q, \Theta)\left(\left\|u^{+}\right\|_{2}+1\right) .
$$

Replacing $u^{+}$with $u^{-}$in all of the above yields the inequality

$$
\sup _{y \in \Theta} u^{-} \leq C(\sigma, q, \Theta)\left(\left\|u^{-}\right\|_{2}+1\right) \text {, }
$$


giving

$$
\|u\|_{L^{\infty}(\Theta)} \leq C(\sigma, q, \Theta,)\left(\|u\|_{2}+1\right) .
$$

To finish the proof we bound $\|u\|_{2}$ independently of $\tau$. Thus, we seek to show that if $u_{\tau}$ is a weak solution to the problem (27), then $\left\|u_{\tau}\right\|_{2} \leq C$ where $C$ is independent of $\tau$. Suppose the inequality is not true. Then $\exists$ a sequence $\left\{\tau_{k}\right\}_{k}$ and a sequence of $Q$-weak solutions $\left\{v_{\tau_{k}}\right\}$ of the Dirichlet problems

$$
\begin{aligned}
L_{\tau_{k}} u & =f \text { in } \Theta \\
u & =0 \text { on } \partial \Theta
\end{aligned}
$$

with $\|f\|_{q / 2}=1\left(q>2 \sigma^{\prime}\right)$ such that $\left\|v_{\tau_{k}}\right\|_{2} \rightarrow \infty$. This forces the sequence $\left\{\tau_{k}\right\}$ to have limit zero. Indeed, if we assume $\tau_{k} \rightarrow \tau>0$ we obtain from (31) that for each $k \in \mathbb{N}$,

$$
\begin{aligned}
\tau_{k}\left\|v_{\tau_{k}}\right\|_{2}^{2} & \leq \mathcal{L}_{\tau}\left(v_{\tau_{k}}, v_{\tau_{k}}\right) \\
& \leq\left|\int_{\Theta} v_{\tau_{k}} f\right| \\
& \leq\left\|v_{\tau_{k}}\right\|_{2} .
\end{aligned}
$$

This yields $\left\|v_{\tau_{k}}\right\|_{2} \leq \frac{1}{\tau_{k}}<\infty$, contradicting $\left\|v_{\tau_{k}}\right\|_{2} \rightarrow \infty$. Thus $\tau_{k} \rightarrow 0$. Set $\beta_{k}=\frac{1}{\left\|v_{\tau_{k}}\right\|_{2}}\left(\right.$ so $\beta_{k} \rightarrow 0$ as $\left.k \rightarrow \infty\right), w_{k}=\beta_{k} v_{\tau_{k}}$ and $f_{k}=\beta_{k} f$. Then $w_{k}$ is a $Q$-weak solution of the Dirichlet problem

$$
\begin{aligned}
L_{\tau_{k}} u & =f_{k} \text { in } \Theta \\
u & =0 \text { on } \partial \Theta .
\end{aligned}
$$

Now, we have that $\left\|w_{k}\right\|_{2}=1$ for each $k$ and so by the compactness of the natural map $I: Q H_{0}^{1}(\Theta) \rightarrow L^{2}(\Theta)$, from Theorem 1.2 we have that there is a subsequence $\left\{w_{k}\right\}_{k=0}^{\infty}$ (relabeled so that we keep the same index) such that $w_{k} \rightarrow w$ in $L^{2}$ and $\|w\|_{2}=1$. Further, we also have $w_{k} \rightarrow w$ in $Q H_{0}^{1}(\Theta)$, giving $w \in Q H_{0}^{1}(\Theta)$ so that we may test $w$ against itself. This is due to the inequality

$$
\begin{aligned}
\int_{\Theta}\left\|\nabla w_{k}\right\|_{Q}^{2} & \leq \mathcal{L}_{\tau_{k}}\left(w_{k}, w_{k}\right) \\
& \leq\left\|w_{k}\right\|_{2}\left\|f_{k}\right\|_{q / 2}=\beta_{k} .
\end{aligned}
$$

Thus we obtain $w$ as a $Q$-weak solution of the Dirichlet problem

$$
\begin{aligned}
L v=\nabla^{\prime} Q(x) \nabla v & =0 \text { in } \Theta \\
v & =0 \text { on } \partial \Theta .
\end{aligned}
$$

By the Poincaré inequality (13) we then obtain that on any ball $B_{r}$ with $r>0$ sufficiently small:

$$
\int_{B_{r}}\left|w-w_{B_{r}}\right|^{2}=0
$$

giving that $w$ is constant on any ball $B_{r}$ with $r>0$ sufficiently small and hence that $w$ is constant in $\Theta$. Without loss of generality we assume $w=1$ in $\Theta$. To obtain a contradiction we now show that (45) implies $w=0$ in $L^{2}(\Theta)$. Let $\Gamma \Subset \Omega$ be an open set so that $\bar{\Theta} \subset \Gamma \subset \bar{\Gamma} \Subset \Omega$. Let $\left\{w_{j}\right\}_{j} \subset \operatorname{Lip}_{0}(\Theta)$ be a sequence representing $w$ in $Q H_{0}^{1}(\Theta)$. Define $\left\{\bar{w}_{j}\right\}_{j} \subset \operatorname{Lip}_{0}(\Gamma)$ where $\bar{w}_{j}=w_{j}$ in $\Theta$ and $\bar{w}_{j}=0$ in $\Gamma \backslash \Theta$. Set $\bar{w}=w$ in $\Theta$ and $w=0$ in $\Gamma \backslash \bar{\Theta}$. Clearly we have that $\bar{w}_{j} \rightarrow \bar{w}$ in $L^{2}(\Gamma)$ and we 
also obtain $\int_{\Gamma} \mathcal{Q}\left(x, \nabla \bar{w}_{j}\right) \rightarrow 0$ as $j \rightarrow \infty$ since $\left(\nabla w_{j}\right)^{\prime} Q(x) \nabla w_{j} \rightarrow(\nabla w)^{\prime} Q(x) \nabla w$ in $L^{1}(\Theta)$. Thus we obtain via the Poincaré inequality that $\bar{w}$ is constant in $\Gamma$. Indeed, from the Poincaré inequality we have that for any ball $B \subset \Gamma$ with radius sufficiently small,

$$
\begin{aligned}
\int_{B}\left|\bar{w}_{j}-\bar{w}_{j_{B}}\right|^{2} & \leq C_{p} r \int_{B}\left(\nabla \bar{w}_{j}\right)^{\prime} Q(x) \nabla \bar{w}_{j} \\
& \leq \int_{\Gamma}\left(\nabla \bar{w}_{j}\right)^{\prime} Q(x) \nabla \bar{w}_{j} \rightarrow 0 \text { as } j \rightarrow \infty .
\end{aligned}
$$

Since $\bar{w}$ is zero in any ball of radius sufficiently small in $\Gamma \backslash \bar{\Theta}$ we then have that $\bar{w}=0$ in $\Gamma$ and hence $w=0$ in $\Theta$, which is a contradiction since $\|w\|_{L^{2}(\Theta)}=1$.

Proof of Theorem 1.1 The proof of this result mimics the proof of the local weak Sobolev inequality (12) in [12, with modifications since we use the global weak Sobolev inequality (11). Set $\eta=\frac{\sigma+1}{2}$; thus $1<\eta<\sigma$. Let $f \in L^{\frac{q}{2}}(\Theta)$; be such that $\|f\|_{\frac{q}{2}}=1$ with $q=2 \eta^{\prime}>2 \sigma^{\prime}$. Let $u$ be the unique weak solution to the Dirichlet problem (27) with $\tau>0$ to be chosen in a moment. Thus, for $v \in \operatorname{Lip}_{0}(\Theta)$ we have:

$$
\begin{aligned}
\int_{\Theta} v^{2} f & =-\int_{\Theta}<\nabla v^{2}, \nabla u>_{Q}-\tau \int_{\Theta} u v^{2} \\
& =-2 \int_{\Theta} v<\nabla v, \nabla u>_{Q}-\tau \int_{\Theta} u v^{2} \\
& \leq 2\left(\int_{\Theta} v^{2}\|\nabla u\|_{Q}^{2}\right)^{1 / 2}\left(\int_{B}\|v\|_{Q}^{2}\right)^{1 / 2}+\tau \int_{\Theta} u v^{2} .
\end{aligned}
$$

Using the fact that $u$ is a weak solution, the square of the first factor satisfies:

$$
\begin{aligned}
\int_{\Theta} v^{2}\|\nabla u\|_{Q}^{2} & =\int_{\Theta}\left(<\nabla\left(u v^{2}\right), \nabla u>_{Q}-2<u \nabla v, v \nabla u>\right) \\
& =-2 \int_{\Theta}<u \nabla v, v \nabla u>_{Q}-\int_{\Theta} u v^{2} f-\tau \int_{\Theta} u^{2} v^{2} \\
\leq & \left(\frac{1}{2} \int_{\Theta} v^{2}\|\nabla u\|_{Q}^{2}+2 \int_{\Theta} u^{2}\|\nabla v\|_{Q}^{2}+\int_{\Theta}|u| v^{2} f+\tau \int_{\Theta} u^{2} v^{2}\right) \\
= & \frac{1}{2} \int_{\Theta} v^{2}\|\nabla u\|_{Q}^{2}+2 \int_{\Theta} u^{2}\|\nabla v\|_{Q}^{2}+\int_{\Theta}|u| v^{2} f+\tau \int_{\Theta} u^{2} v^{2} .
\end{aligned}
$$

Absorbing the first term on the right into the left hand side and multiplying by 2 yields:

$$
\begin{gathered}
\int_{\Theta} v^{2}\|\nabla u\|_{Q}^{2} \leq 4\left(\sup _{\Theta}|u|\right)^{2} \int_{\Theta}\|\nabla v\|_{Q}^{2}+2\left(\sup _{\Theta}|u|\right) \int_{\Theta} v^{2} f+2 \tau \int_{\Theta} u^{2} v^{2} \\
\leq 4 \max \left\{\left(\sup _{\Theta}|u|\right)^{2} \int_{\Theta}\|\nabla v\|_{Q}^{2},\left(\sup _{\Theta}|u|\right) \int_{\Theta} v^{2} f,\left(\sup _{\Theta}|u|\right)^{2} \tau \int_{\Theta} v^{2}\right\} .
\end{gathered}
$$


No matter which term on the right is the maximum, (48) with (29) gives:

$$
\begin{aligned}
\int_{B} v^{2} f & \leq C\left\{\left(\sup _{\Theta}|u|\right) \int_{\Theta}\|\nabla v\|_{Q}^{2}+\tau\left(\sup _{\Theta}|u|\right) \int_{\Theta} v^{2}\right\} \\
& \leq C|\Theta|^{\frac{2}{q}}\left\{\left(\frac{1}{|\Theta|} \int_{\Theta}|f|^{\frac{q}{2}}\right)^{\frac{2}{q}} \int_{\Theta}\|\nabla v\|_{Q}^{2}+\tau\left(\frac{1}{|\Theta|} \int_{\Theta}|f|^{\frac{q}{2}}\right)^{\frac{2}{q}} \int_{\Theta} v^{2}\right\} \\
= & C|\Theta|^{\frac{1}{\eta^{\prime}}}\left\{\left(\frac{1}{|\Theta|} \int_{\Theta}|f|^{\eta^{\prime}}\right)^{\frac{1}{\eta^{\prime}}} \int_{\Theta}\|\nabla v\|_{Q}^{2}+\tau\left(\frac{1}{|\Theta|} \int_{\Theta}|f|^{\eta^{\prime}}\right)^{\frac{1}{\eta^{\prime}}} \int_{\Theta} v^{2}\right\} .
\end{aligned}
$$

Thus, we have:

$$
\begin{aligned}
\left(\frac{1}{|\Theta|} \int_{\Theta}|v|^{2 \eta}\right)^{\frac{1}{\eta}} & =\sup _{\frac{1}{|\Theta|} \int_{\Theta}|f|^{\eta^{\prime}}=1}\left|\frac{1}{|\Theta|} \int_{\Theta} v^{2} f\right| \\
& \leq C|\Theta|^{\frac{1}{\eta^{\prime}}}\left\{\left(\frac{1}{|\Theta|} \int_{\Theta}\|\nabla v\|_{Q}^{2}+\tau \int_{\Theta} v^{2}\right)\right\} .
\end{aligned}
$$

Taking square roots of both sides gives:

$$
\left(\frac{1}{|\Theta|} \int_{\Theta}|v|^{2 \eta}\right)^{\frac{1}{2 \eta}} \leq C\left\{\left(\int_{\Theta}\|\nabla v\|_{Q}^{2}\right)^{\frac{1}{2}}+\tau^{\frac{1}{2}}\left(\int_{\Theta} v^{2}\right)^{\frac{1}{2}}\right\} .
$$

At this point we are free to choose $\tau$ as close to zero as we like. We choose $\tau>0$ sufficiently small so that we may absorb the $L^{2}$ norm on the right hand side into the $L^{2 \eta}$ norm on the left. We are able to do this since $2 \leq 2 \eta$. This yields the Sobolev inequality

$$
\left(\frac{1}{|\Theta|} \int_{\Theta}|v|^{2 \eta}\right)^{\frac{1}{2 \eta}} \leq C\left(\frac{1}{|\Theta|} \int_{\Theta}\|\nabla v\|_{Q}^{2}\right)^{\frac{1}{2}}
$$

holding for all $v$. Combining this result with the weak Sobolev inequality on $\Theta$, (26), we obtain:

$$
\begin{aligned}
\left(\int_{\Theta}|w|^{2 \sigma}\right)^{\frac{1}{2 \sigma}} & \leq C\left\{\left(\int_{\Theta} \mathcal{Q}(x, \nabla w)\right)^{\frac{1}{2}}+\tilde{C}\left(\int_{\Theta}|w|^{2 \eta}\right)^{\frac{1}{2 \eta}}\right\} \\
& \leq C\left(\int_{\Theta} \mathcal{Q}(x, \nabla w)\right)^{\frac{1}{2}},
\end{aligned}
$$

for $w \in \operatorname{Lip}_{0}(\Theta)$.

\section{REFERENCES}

1. Eugene B. Fabes, Carlos E. Kenig, and Raul P. Serapioni, The local regularity of solutions of degenerate elliptic equations, Comm. Partial Differential Equations 7, no. 1 (1982), 77-116. MR643158 (84i:35070)

2. C. Fefferman and D.H. Phong, The local regularity of solutions to degenerate elliptic equations, Comm. Partial Differential Equations 7 (1982), 77-116. MR643158 (84i:35070)

3. B. Franchi, R. Serapioni, and F. Serra Cassano, Approximation and imbedding theorems for weighted Sobolev spaces associated with Lipschitz continuous vector fields, Boll. Unione Mat. Ital. 7, 11-B (1997), 83-117. MR.1448000 (98c:46062)

4. David Gilbarg and Neil S. Trudinger, Elliptic partial differential equations of second order, Springer, 1998.

5. C.E. Gutierrez and E. Lanconelli, Maximum principle, non-homogeneous Harnack inequality, and Liouville theorems for x-elliptic operators, Comm. Partial Differential Equations 28, no. 11-12 (2003), 1833-1862. MR2015404 (2004j:35030)

6. Peter D. Lax, Functional analysis, Wiley-Interscience, New York, 2002. MR.1892228 (2003a:47001) 
7. Scott Rodney, Existence of weak solutions to subelliptic partial differential equations in divergence form and the necessity of the Sobolev and Poincaré inequalities, Ph.D. thesis, McMaster University, Hamilton, Ontario, Canada, 2007.

8. H.L. Royden, Real analysis, third edition, Prentice-Hall, 1988. MR 1013117 (90g:00004)

9. Walter Rudin, Principles of mathematical analysis, McGraw Hill, Boston, Massachusetts, 1976. MR0385023 (52:5893)

10. _ Real and complex analysis, McGraw Hill, Boston, Massachusetts, 1987. MR.924157 (88k:00002)

11. Eric Sawyer and Richard L. Wheeden, Weighted inequalities for fractional integrals on Euclidean and homogeneous spaces, Amer. J. Math. 114 (1992), 813-874. MR.1175693 (94i:42024)

12. Hölder continuity of weak solutions to equations with rough coefficients, Mem. Amer. Math. Soc. 847 (2006). MR2204824 (2007f:35037)

13. Eric T. Sawyer and Richard L. Wheeden, Degenerate Sobolev spaces and weak solutions, Trans. Amer. Math. Soc., to appear.

14. Leon Simon, Lectures on geometric measure theory, Proceedings of the Centre for Mathematical Analysis, Australian National University 3 (1983). MR756417(87a:49001)

15. Elias M. Stein, Harmonic analysis, Princeton University Press, 1993. MR.1232192 (95c:42002)

Department of Mathematics, Rutgers University, Piscataway, New Jersey 08854 